IMPROVING CUBESAT DOWNLINK CAPACITY WITH

ACTIVE PHASED ARRAY ANTENNAS

By

Jonathan Klein, B.S.

A Project Submitted in Partial Fulfillment of the Requirements

for the Degree of

Master of Science

in

Electrical Engineering

University of Alaska Fairbanks

August 2017

APPROVED:

Joe Hawkins, Committee Chair

Denise Thorsen, Co-Chair

Dejan Raskovic, Committee Member Charlie Mayer, Chair

Department of Electrical and Computer Engineering 


\section{Abstract:}

Power budgets on small satellites are restricted by the limited surface area for solar panels. This limits the power available for radio communications, which constrains the downlink budget. The limited transmit power translates to low downlink data rates on small satellites. Antenna gain from directive antennas may be a power efficient way of improving the downlink budget, thereby increasing the downlink rate of small satellites.

This project focuses on the design and development of a prototype low-power, electrically-steered S-band phased array RF front-end suitable for a CubeSat that could efficiently increase the EIRP, permitting higher data rates. A prototype of the array has been constructed and tested in an anechoic chamber. The four element array provides a minimum gain of $2.5 \mathrm{~dB}$ and average gain of $5 \mathrm{~dB}$ compared to a single patch antenna element with a 5W power envelope across a range of up to 60 degrees from broadside of the array. 


\title{
Improving CubeSat Downlink Capacity with Active Phased Array Antennas
}

\author{
Jonathan Klein, Joe Hawkins, Denise Thorsen \\ Department of Electrical and Computer Engineering \\ University of Alaska, Fairbanks \\ Fairbanks, AK 99775 \\ 907-474-7613 \\ jtklein@alaska.edu,jghawkins@alaska.edu, dlthorsen@alaska.edu
}

\begin{abstract}
Power budgets on small satellites are restricted by the limited surface area for solar panels. This limits the power available for radio communications, which constrains the downlink budget. The limited transmit power translates to low downlink data rates on small satellites. Antenna gain from directive antennas may be a power efficient way of improving the downlink budget, thereby increasing the downlink rate of small satellites. We have developed a prototype low-power, electrically-steered S-band phased array RF front-end suitable for a CubeSat that could efficiently increase the EIRP, permitting higher data rates. A prototype of the array has been constructed and tested in an anechoic chamber. The four element array provides a minimum gain of $2.5 \mathrm{~dB}$ and average gain of $5 \mathrm{~dB}$ compared to a single patch antenna element with a $5 \mathrm{~W}$ power envelope across a range of up to 60 degrees from broadside of the array. Given the $100 \mathrm{~mW}$ overhead of each phased array element, the design is expected to scale to a 16 element array, which could fit on two $20 \mathrm{~cm}$ by $10 \mathrm{~cm}$ deployable panels on a $2 \mathrm{U}$ CubeSat. With a 10 watt average power budget for the transmitter, such an array would provide an estimated gain of $11 \mathrm{~dB}$ compared to a single omnidirectional antenna.
\end{abstract}

\section{Table of Contents}

1 InTRODUCTION ...............................

2 Phased array Concept and Hardware

DESIGN ............................... 1

3 Testing and Performance Validation .... 5

4 Conclusion ............................... 7

ACKNOWLEDGMENTS ....................... 7

REFERENCES .............................. 7

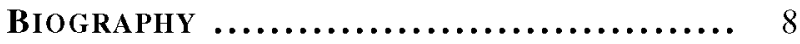

\section{INTRODUCTION}

The downlink budget is a bottleneck to communications on nanosatellites such as CubeSats. Transmit signal power from many CubeSats is constrained by the limited surface area for solar panels, and antenna gain is limited by use of omnidirectional antennas. Additionally, CubeSats are frequently constructed and operated by universities without access to large high gain dishes capable of tracking LEO satellites. While off-the-shelf commercial radios designed for CubeSats are available supporting data rates in excess of $1 \mathrm{Mbps}$, only a minority of CubeSats achieve these speeds [1] [2] [4] [5]. Transmitting at high data rates is possible, but it is difficult to achieve a sufficient signal-to-noise ratio to successfully decode these transmissions.

978-1-4799-1622-1/14 (C)2014 IEEE.

${ }^{1}$ IEEEAC Paper \#2140, Version 11, Updated 12/30/2013.
One approach to improving the link budget of CubeSats could be to increase the satellite's antenna gain. Adding antenna gain using directional antennas reduces the transmitted power required to maintain a link budget. However, directional antennas must be pointed at a ground station to be effective. Antennas could be steered mechanically by moving the array or reorienting the satellite, or electrically with phased arrays. Electrical steering using phased arrays is potentially more power efficient than mechanical steering and may be preferred if reorienting the satellite would conflict with other systems such as cameras or solar panels, or if the satellite lacks precise attitude control.

Phased arrays have the potential to increase the power efficiency of link budgets compared to omnidirectional antennas provided that the DC power consumed by controlling the array is not greater than the decrease in transmitted power made possible by antenna gain. A more efficient downlink could increase the downlink rate and return more data on each pass or leave more power for the satellite payload. However, nearly all CubeSats use single patch antennas for microwave downlinks, and information about phased arrays for CubeSats is sparse. A University of Hawaii Mea Huaka ' 1 CubeSat included a 5.8-GHz phased array, however its launch vehicle failed and it was not tested in orbit [5]. The University of Hawaii has also designed compact retrodirective array prototypes, however their design consumed too much power to be feasible to implement on CubeSats [3]. Lockheed Martin mentions the Phased Array Tile Rapid In-Orbit Test (PATRIOT) CubeSat concept in marketing literature, however they do not publicly mention if the proposed satellite was constructed [6].

This paper presents a prototype hardware design for a CubeSat-scale phased array and analyzes the performance of the array from the perspective of increasing the link budget with fixed power consumption. This paper focuses on design requirements for a phased array, the RF hardware design, array calibration, and performance validation.

\section{Phased array Concept and HARDWARE DESIGN}

The goal of the phased array is to maximize equivalent isotropically radiated power (EIRP) with a fixed power budget. Each element in the phased array adds overhead, which diminishes the marginal return to the link budget of adding elements to the array. A maximum useful range of array sizes exists as a function of the efficiency of the array elements. The EIRP at the mainlobe of an $N$ element array with isotropic antennas and $\frac{\lambda}{2}$ antenna spacing can be modeled as:

$$
E I R P=\eta_{a m p}\left(P_{\text {tot }}-N P_{\text {elem }}\right) N
$$


where $P_{\text {tot }}$ is the total power budget for the array, $P_{\text {elem }}$ is the per element overhead, and $\eta_{a m p}$ is the transmit amplifier power added efficiency. The $P_{\text {elem }}$ per-element overhead factor describes the DC power used by $\mathrm{T} / \mathrm{R}$ modules that is not consumed by the transmit power amplifier. Figure 1 illustrates Equation 1 for $\eta_{a m p}=25 \%$ and $P_{\text {elem }}=100 \mathrm{~mW}$.

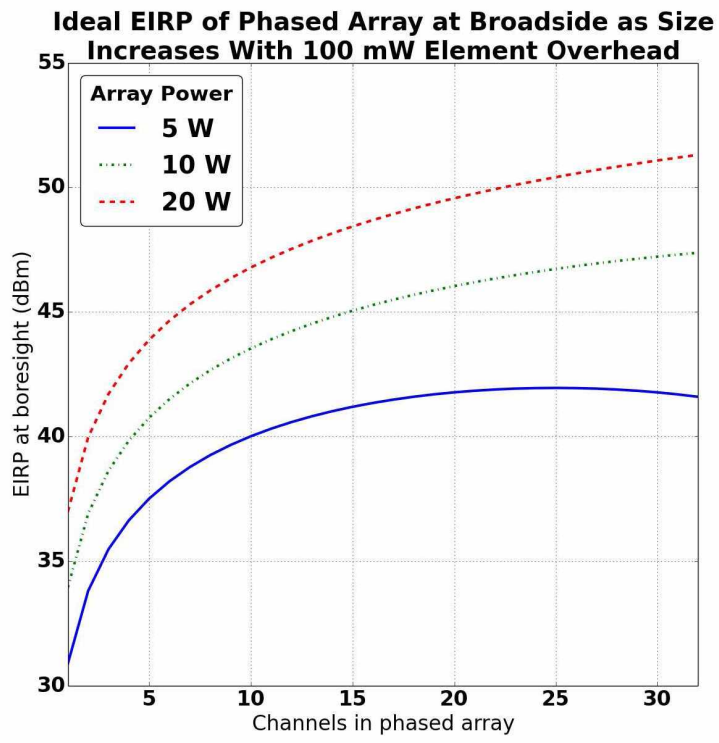

Figure 1. EIRP versus size of phased array for power budgets of $5 \mathrm{~W}, 10 \mathrm{~W}$, and $20 \mathrm{~W}$.

Note that with $P_{\text {tot }}$ equal to $5 \mathrm{~W}$, the EIRP of the array in Figure 1 does not benefit from adding more than 25 channels to the phased array. This simple model does not take into account element spacing, losses from mutual coupling, losses due to imperfect beamforming, or the radiation pattern of the antenna elements. A multiplier $\eta_{\text {array }}$ could be added to account for losses due to mutual coupling and imperfect phasing. Modeling the directivity of planar arrays with variable element spacing and antenna radiation patterns is developed in several papers and books [7] [9]. Adding the gain of an array $G_{a r r a y}(\theta, \phi)$, which can be calculated as a function of the individual element radiation pattern and the element-to-element spacing, the EIRP of the phased array could be modeled with the following equation:

$$
E I R P=\eta_{a m p} G_{\text {array }}(\theta, \phi)\left(P_{\text {tot }}-N P_{\text {elem }}\right) \eta_{\text {array }}
$$

This equation can be used to evaluate configurations of the prototype CubeSat phased array design. A two element by eight element array using patch antennas described later in the paper with $.42 \lambda$ spacing is expected to increase antenna gain by $12 \mathrm{~dB}$ relative to a single element at broadside. This increase in antenna gain from the array is calculated numerically from a measured radiation pattern of a single T/R module from the array. Using $P_{\text {tot }}=10 \mathrm{~W}, \eta_{a m p}=$ $25 \%$, Garray $=12 \mathrm{~dB}$, and $P_{\text {elem }}=100 \mathrm{~mW}$, a 16 element $\mathrm{S}$-band array is predicted by Equation 2 to provide $11.3 \mathrm{~dB}$ increase in EIRP over a single antenna element after accounting for DC power consumption of the array. Measured error in the phase shifters results in a $\eta_{a m p}$ of $-0.3 \mathrm{~dB}$. Combining the $.7 \mathrm{~dB}$ loss from the $1.6 \mathrm{~W}$ array overhead with $.3 \mathrm{~dB}$ loss
Table 1. 2U Phased Array Size with Frequency using $.42 \lambda$ Element Spacing and Patch Antennas

\begin{tabular}{|c|c|c|}
\hline Frequency & $\lambda$ & Elements \\
\hline \hline $144 \mathrm{MHz}$ & $200 \mathrm{~cm}$ & 0 \\
$440 \mathrm{MHz}$ & $70 \mathrm{~cm}$ & 0 \\
$900 \mathrm{MHz}$ & $33 \mathrm{~cm}$ & 0 \\
$1.2 \mathrm{GHz}$ & $25 \mathrm{~cm}$ & 2 \\
$2.48 \mathrm{GHz}$ & $12 \mathrm{~cm}$ & 8 \\
$5.8 \mathrm{GHz}$ & $5.2 \mathrm{~cm}$ & 36 \\
$10.1 \mathrm{GHz}$ & $2.9 \mathrm{~cm}$ & 128 \\
\hline
\end{tabular}

from phase shifter errors, a 16 element array is expected to result in a $11 \mathrm{~dB}$ gain relative to a single antenna element at broadside with a $10 \mathrm{~W}$ power budget.

Not all frequencies and array topologies work well with nanosatellites. The surface area on CubeSats limits which transmit frequencies are feasible. Phased arrays require element-to-element spacing at some moderate fraction of a wavelength. This means the UHF and VHF frequencies commonly used by CubeSats will not work without long deployable elements. Table 1 shows a comparison of possible patch antenna phased array sizes at possible CubeSat downlink frequencies with a $2 \mathrm{U}(20 \mathrm{~cm}$ by $10 \mathrm{~cm})$ panel and $.42 \lambda$ spacing.

The $2.4 \mathrm{GHz}$ band was selected for the prototype phased array as the lowest practical frequency where it is possible to fit multiple elements on a $1 \mathrm{U}$ face with azimuth/elevation steering. It was chosen over the $5.8 \mathrm{GHz}$ and $10 \mathrm{GHz}$ bands because of availability of off-the-shelf MMIC components.

The low power budgets on CubeSats constrains possible phased array designs. Some phased array topologies are not power efficient enough to provide an improvement to the downlink budget. The overhead from power hungry phase shifting techniques (such as I/Q modulation of each channel with a DDS and mixers) consume the benefits of increased antenna gain. Placing a MMIC phase shifter before a power amplifier was determined to be the most efficient configuration. This topology uses negligible power to create a phase shift and minimizes losses to the signal at high power levels.

The phased array prototype works as an RF front end; it needs a radio transceiver to synthesize an RF signal and to process received signals from the T/R modules. The array divides the transmitted signal into multiple channels and then phase shifts and amplifies each channel individually. See Figure 2 for a block diagram of the phased array design.

The hardware design of the phased array focused on the T/R modules, control bus, and feed network. The receive path is provided for future development of a retrodirective antenna and is not discussed in this paper.

\section{T/R Module}

The T/R modules provide a phase shift and amplification of the transmitted signal, and amplification of the received signal. Four T/R modules were designed and fabricated for the phased array. See Figure 3 for a block diagram of the T/R modules, and Figures 4 and 5 for photographs of a module prototype. 


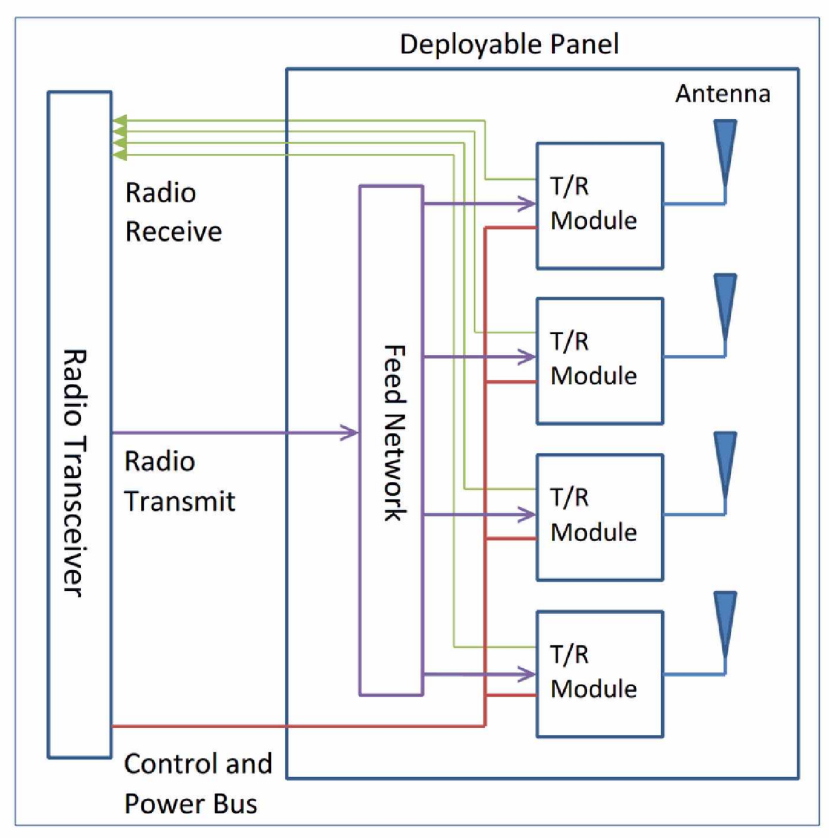

Figure 2. Block diagram of the phased array. Green arrows indicate the receive signal path, purple arrows the transmitted signal path, and red arrows are the control and power bus.

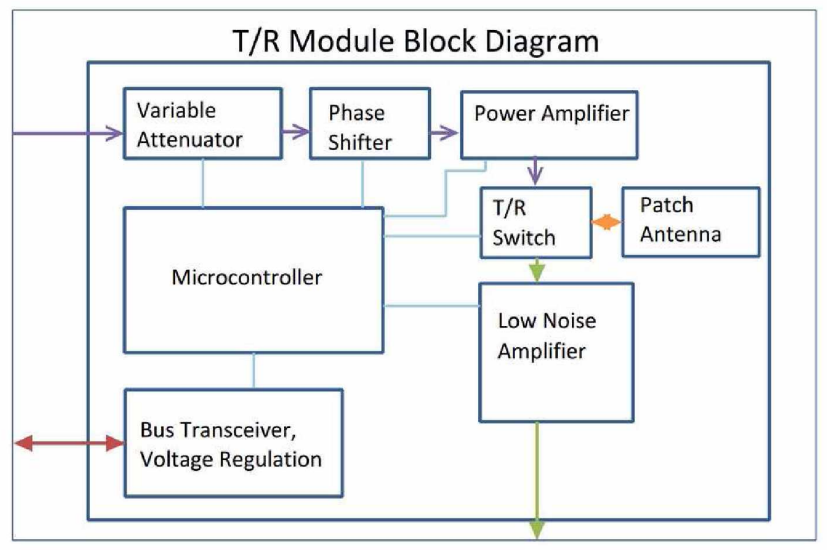

Figure 3. Block diagram of a $T / R$ module.

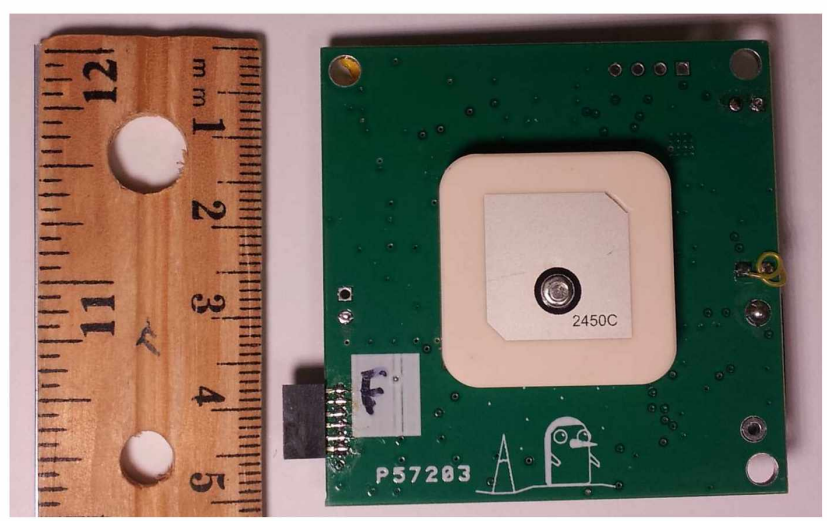

Figure 4. Photograph of the top side of a T/R module.

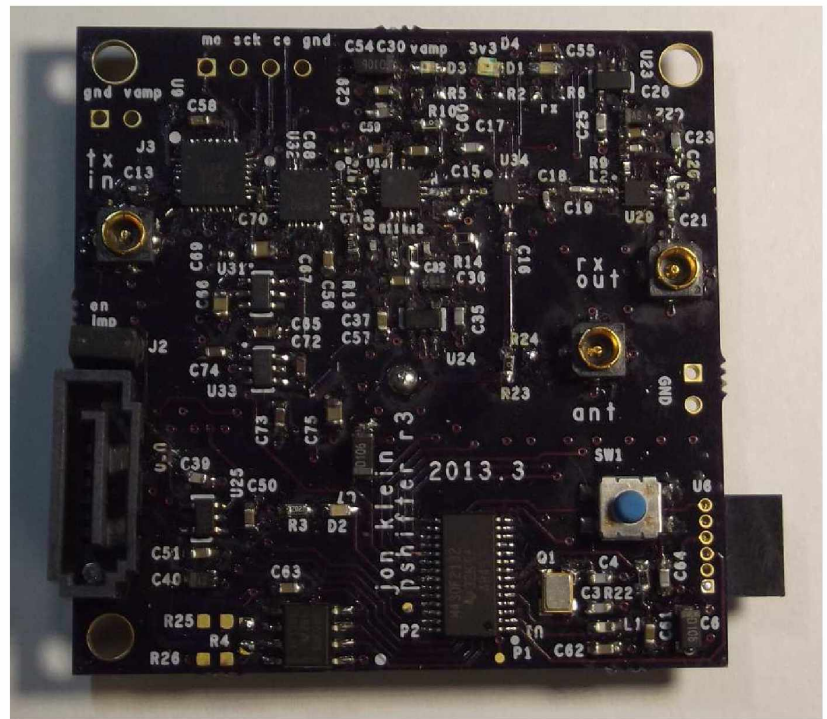

Figure 5. Photograph of the bottom side of a T/R module.

Power consumed by the T/R modules that is not used by the transmit power amplifiers is considered overhead (the efficiency of the power amplifiers is accounted for separately). This includes the communications bus, microcontrollers, phase shifters, and variable attenuators which would not be needed on a single channel system. A power overhead of $100 \mathrm{~mW}$ per module while in transmit mode accounts for the overhead of the T/R modules. This figure was calculated by summing the power consumption of each of the components on the T/R module other than the transmit power amplifier. To minimize this overhead the modules of some components in the transmit and receive signal paths can be disabled when not in use.

The transmit path of the phased array modules provides gain with variable attenuation and phase shift to the input signal from the feed network. The signal is first attenuated through a Skyworks SKY12343 7-bit digital attenuator, then phase shifted with a M/A-COM MAPS-010164 6-bit digital phase shifter. The variable attenuator is used to compensate for attenuation variations in the phase shifter as a function of phase shift, and to equalize the gain of the power amplifiers between channels. The signal is attenuated before the power amplifier to minimize the loss in efficiency and to provide a consistent input power level into the transmit power amplifier The attenuator adds less than $5 \mathrm{~mW}$ to the overhead of each module and it allows the transmit power amplifier to be kept at a constant output power level. A Microchip SST12LP15B $2.4 \mathrm{GHz}$ power amplifier provides gain for a $24 \mathrm{dBm}$ output power per channel at the antenna with $3 \mathrm{dBm}$ input into the T/R module. Another amplifier could be used if a different output power level or lower distortion is needed. The array is not capable of simultaneous transmit and receive; when the module is not in transmit mode the phase shifter and power amplifier are disabled to reduce power consumption.

The receive path switches and amplifies signals from the antenna to a receive port on the module. To reduce the complexity of the T/R module, the received signal is not routed through the phase shifter. Any beamforming of the received signal must be done in software by simultaneously sampling multiple channels. 


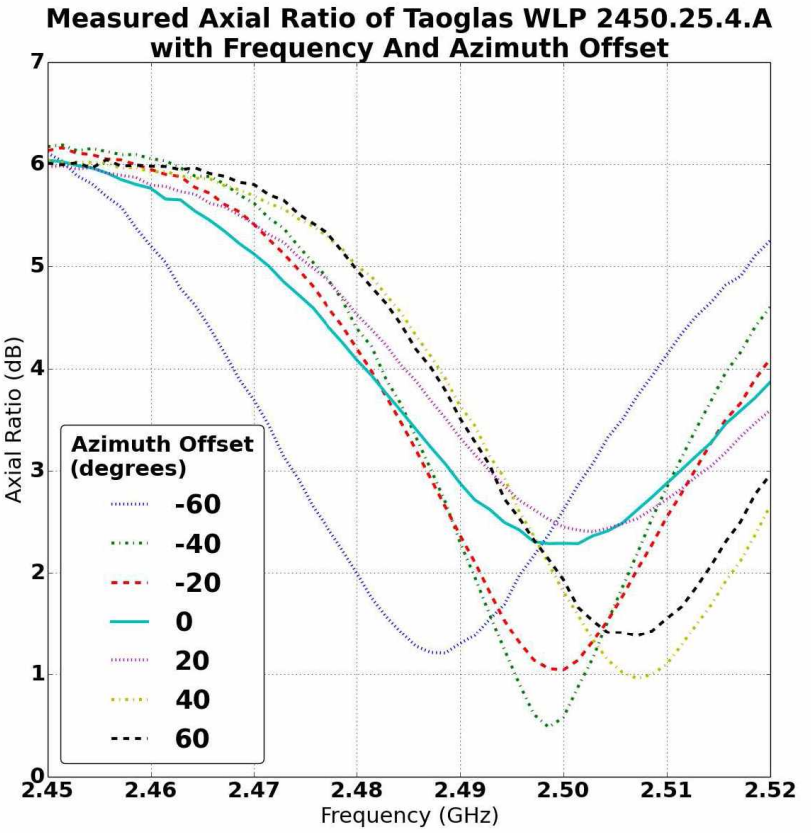

Figure 6. Measured axial ratio versus frequency of the Taoglas WLP.2450.25.4.A.02 with azimuth offset.

Direction finding algorithms could aid in steering the array by determining the location of the ground station transmitting a beacon tone by processing simultaneous samples from the array. If beamforming or switching between channels is not necessary, the receive path from one channel could be connected to the radio receiver and the remaining channels could be disabled or depopulated. The Skyworks 67014 LNA is switched off to reduce power consumption when the module is not in receive mode.

The T/R modules integrate a patch antenna on the reverse side as seen in Figure 4. Circularly polarized antennas were used to minimize polarization loss as CubeSats may not have control of their orientation relative to a ground station. The Taoglas WLP.2450.25.4.A.02 right-hand circularly polarized (RHCP) ceramic patch is used in the current prototype. This antenna is only circularly polarized over a narrow bandwidth. As shown in Figure 6, the measured bandwidth of the patch with an axial ratio below $3 \mathrm{~dB}$ is approximately $0.3 \%$ up to a 60 degree azimuth offset. The antenna may need redesign after a specific frequency has been allocated for a satellite mission.

See Table 2 for an overview of the performance of the T/R modules.

\section{Feed Network}

A feed network is necessary to distribute the transmitted signal to phased array modules. Variations in the phase and gain between different paths in the feed network are compensated for by the phase shifter and attenuator using lookup tables to determine the settings for each channel, so the feed network does not need equal path lengths to each element. The ZA4PD-4 4-way 0-degree power splitter from Mini-Circuits is used in the current prototype. A smaller feed network could be constructed to fit the mechanical requirements of a specific satellite with microstrip circuits or surface mount power splitters.
Table 2. Phased Array T/R Module Specifications

\begin{tabular}{|c|c|c|}
\hline Parameter & Min & Max \\
\hline \hline Phase Shift Range & $0^{\circ}$ & $360^{\circ}$ \\
Gain (TX Path) & $-4 \mathrm{~dB}$ & $24 \mathrm{~dB}$ \\
Gain (RX Path) & $9 \mathrm{~dB}$ & $11 \mathrm{~dB}$ \\
Phase Error & - & $4.5^{\circ} \mathrm{RMS}$ \\
Amplitude Error & - & $0.3 \mathrm{~dB} \mathrm{RMS}$ \\
PAE at 24 dBm & $25 \%$ & - \\
Standby Mode Power & - & $55 \mathrm{~mW}$ \\
Receive Mode Power & - & $85 \mathrm{~mW}$ \\
Transmit Mode Power & - & $1800 \mathrm{~mW}$ \\
Element Power Overhead & - & $100 \mathrm{~mW}$ \\
Frequency & $2400 \mathrm{MHz}$ & $2500 \mathrm{MHz}$ \\
\hline
\end{tabular}

\section{Calibration}

Ideally no phase variation would exist between phased array channels other than differences programmed by the phased array controller for beamforming. Likewise, in the absence of weighting, there would ideally be no variation in amplitude between channels in the array. In reality, there are unintended amplitude and phase offsets between channels in the array from several sources. These phase and amplitude variations must be measured to calibrate the array and evaluate its performance.

There are several sources of phase error within the array. The largest contributors are path length differences in the feed network, attenuation dependent phase shifts, and differences in the power amplifier matching network between T/R module revisions. The electrical length from the power splitter to the $\mathrm{T} / \mathrm{R}$ modules is not equal in the current prototype as longer cables were needed to reach some elements. This introduces a frequency dependent phase shift to those channels which would create an elevation angle offset to the beam if left uncompensated. Amplitude also varies between channels; up to $3.5 \mathrm{~dB}$ in amplitude variation is expected from the gain of the power amplifier and insertion losses of the phase shifter, attenuator, and T/R switch.

To compensate for differences, the amplitude and phase response of the T/R modules is measured on a network analyzer. The calibration plane of these measurements extends to the input of the feed network at one port and an RF connector near the antenna for the other port. This measures differences in path length of the feed network as well as amplitude and phase variations in the T/R modules. The Sparameters of the transmit paths were measured and recorded on a computer at all 64 phase shifter states and the first 20 variable attenuator states using a script. Data from these measurements are used to generate calibration lookup tables. Calibration measurements are currently taken at an uncontrolled room temperature and the array has not yet been tested in a temperature controlled chamber. Additional calibration tables across temperature variations may be required.

Phase and amplitude variations are corrected with calibration tables. To generate calibration tables at a single frequency, the lowest error combination of phase shifter and attenuator states is computed for a fixed amplitude and 180 phase steps. The output amplitude for the T/R modules is selected to balance amplifier efficiency and signal distortion. 

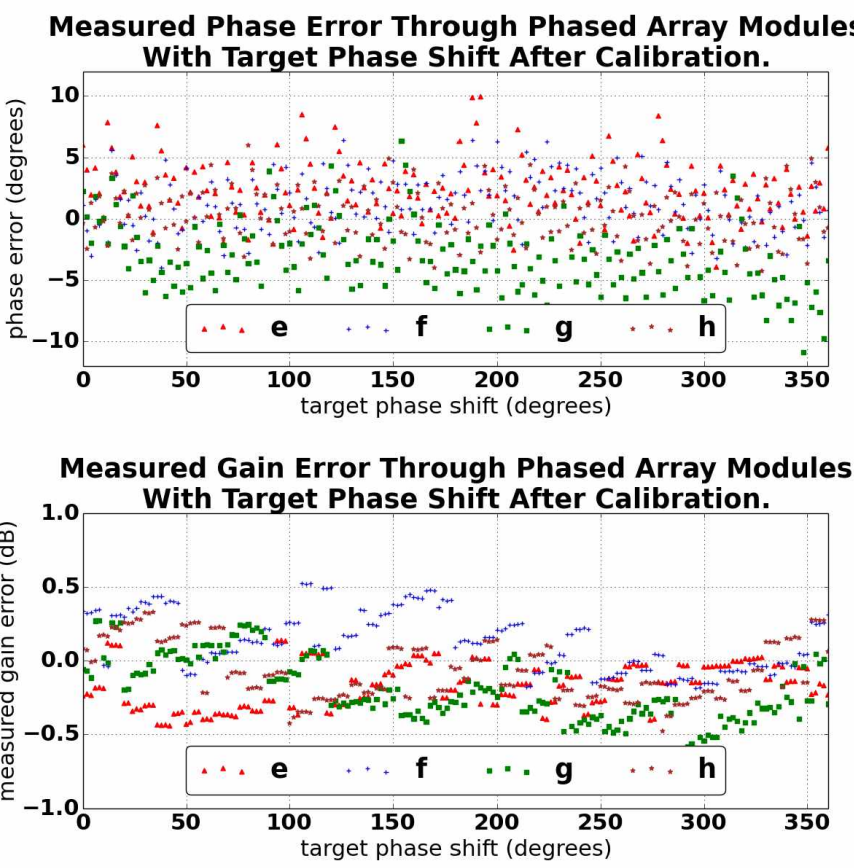

Figure 7. Measured phase and amplitude error through each of the four T/R modules (labeled e, f, g, and h) following calibration.

First, the lowest error phase shifter state is calculated for each phase step. Then, the lowest error attenuation setting to set the power at the antenna port to a predetermined value is determined. The phase shifter register settings are then recomputed to account for the variations in phase shift from the attenuation. The final phase shifter and attenuator register values for each phase offset step are stored in non-volatile memory on the phased array module.

Following calibration the phase and amplitude error of the T/R modules is measured. This is done by stepping the modules through phase settings at a calibrated fixed output power and comparing the measured phase shift with the desired phase setting. The difference between the measured and intended phase shift is the phase error, and the difference between the measured gain and expected gain is the gain error. See Figure 7 for a plot of the measured phase and amplitude error through the four prototype T/R modules and feed network following calibration. The highest RMS phase error following calibration is 4.5 degrees across all phase shift steps of the four prototype T/R modules. This is comparable to the 3 degree RMS quantization error specified by the manufacturer of the phase shifter chip. A 4.5 degree RMS phase error is calculated to degrade the output SNR of the phased array prototype by $.3 \mathrm{~dB}$ [8]. The amplitude error following calibration is $.29 \mathrm{~dB}$ RMS, which is near the .26 $\mathrm{dB}$ quoted in the datasheet of the variable attenuator.

\section{Testing and Performance Validation}

The functionality and performance of the phased array was validated in an anechoic chamber. Vector network analyzer (VNA) $S_{21}$ measurements representing the path loss through the anechoic chamber are used to measure the relative gain of the array. These measurements are taken with the VNA calibration plane extending to the input of the feed network at one port and the connector of the receiving antenna at the other port. The antenna gain of the phased array is computed from these measurements by comparing the $S_{21}$ measurements from a single antenna to the $S_{21}$ measurements of the array at varying electrical steering and azimuth and elevation orientations.

The radiation pattern is measured by mounting the array to a platform with adjustable azimuth and elevation angle. This platform was constructed with servo gearboxes and plastic components fabricated with a 3D printer. See Figure 8 for a picture of the phased array showing the feed network, control bus, and the elevation servo. See Figure 9 for a picture of the front of the four element phased array prototype in an anechoic chamber.

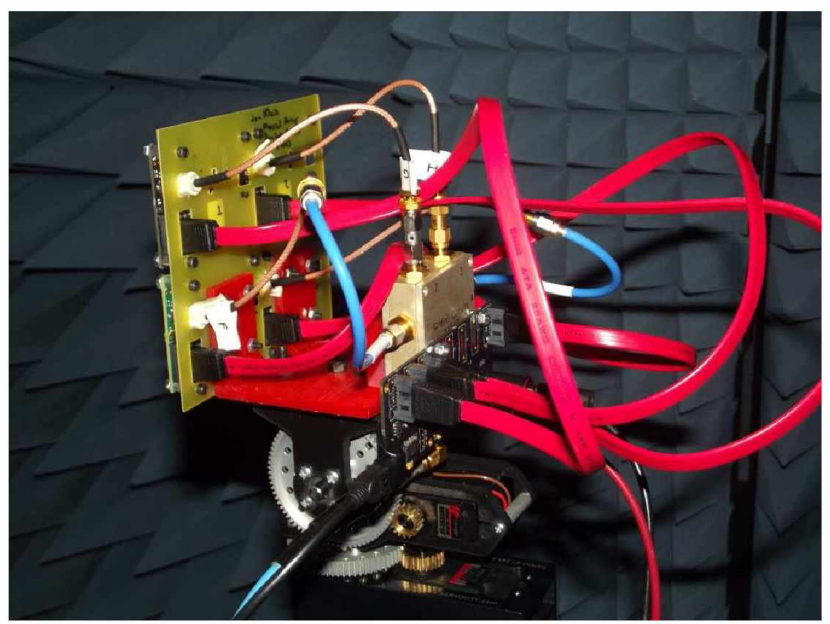

Figure 8. Photograph back side of the phased array inside an anechoic chamber.

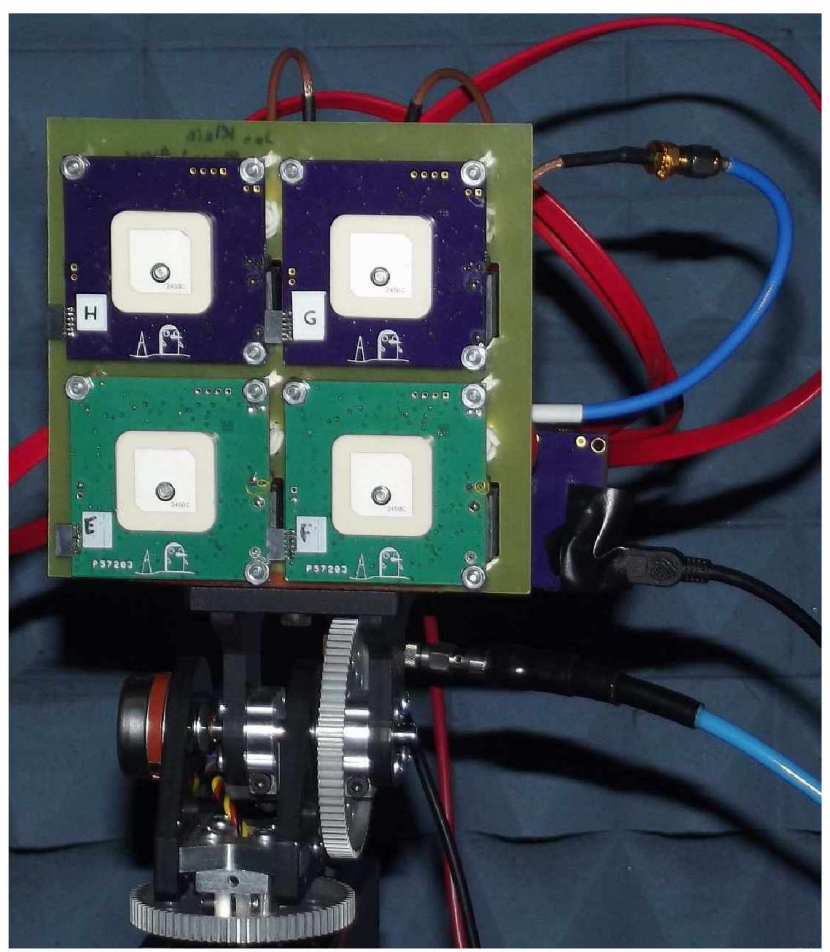

Figure 9. Photograph of the phased array prototype mounted on a tripod inside an anechoic chamber. 
The effectiveness of the phased array is determined by calculating the gain of the transmitting phased array compared to a single element with a receiving RHCP antenna. Polarization of the signal is measured by transmitting into a rotating linearly polarized antenna. The axial ratio and received signal power is calculated by fitting an ellipse to $\left|S_{21}\right|$ measurements taken while rotating the linearly polarized receive antenna. Polarization mismatch loss to a circularly polarized antenna is then calculated from these measurements with the following equation, where $\gamma_{e}$ is the measured axial ratio [10].

$$
\eta_{\text {mismatch }}=10 \log _{10}\left(\frac{1}{2}+\frac{\gamma_{\mathrm{e}}}{1+\gamma_{\mathrm{e}}^{2}}\right)
$$

The gain from the phased array is calculated by comparing path loss measurements across an anechoic chamber of a single T/R module with an array of T/R modules. The phased array $T / R$ modules are calibrated to a fixed output power level. Therefore, quadrupling the number of transmitting modules increases the EIRP of the phased array by $6 \mathrm{~dB}$ independent of the antenna gain. Additionally, a fraction of the phased array power budget is the DC power overhead from the array. The phased array path loss measurements are compensated to account for the overhead of the phased array modules and the increased transmit power from multiple active elements. This can be calculated as a function of the element power $P_{\text {elem }}$, the total power $P_{t o t}$, and the number of elements $N$. The following equation describes the correction to path loss applied to anechoic chamber array measurements to compare them with individual element measurements for $N$ elements, a total power budget of $P_{t o t}$, and an element overhead of $P_{\text {elem }}$.

$$
\Delta \mathrm{P}_{\text {loss }}=10 \log _{10}(\mathrm{~N})-10 \log _{10}\left(\frac{\mathrm{P}_{\text {tot }}-\mathrm{NP}_{\text {elem }}}{\mathrm{P}_{\text {tot }}}\right)
$$

For the 4 element phased array prototype with a 5 watt power budget and a $100 \mathrm{~mW}$ per-element overhead, an additional $6.4 \mathrm{~dB}$ of path loss is added to the array measurements when comparing them to the individual element path loss measurements. The calculated gain from the phased array is the difference in magnitude between the path loss through the anechoic chamber of a single element and the compensated path loss of the array electrically steered to point at the receiving antenna.

\section{Test Results}

Measurements show the four element phased array in a two element by two element planar configuration increases antenna gain compared to a single patch antenna element. The radiation pattern of the phased array was compared to an expected radiation pattern calculated from measurements of an individual $\mathrm{T} / \mathrm{R}$ module. The expected radiation pattern eliminates the effects of phase quantization and mutual coupling. If the measured and expected radiation patterns closely match, it would indicate that mutual coupling and quantization effects are minimal. Phase shifts for the expected radiation pattern are applied in software to remove any phase quantization effects. Other T/R modules were disconnected and detached from the array when measuring the individual element radiation pattern to eliminate mutual coupling. Figure 10 shows the array electrically steered to 30 degrees on the azimuth axis and 30 degrees on the elevation

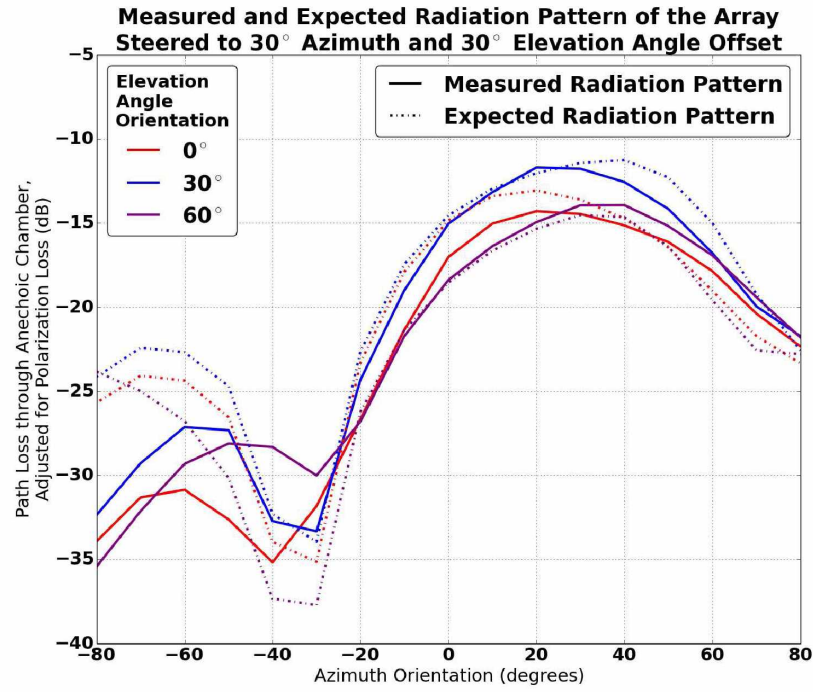

Figure 10. Measured and calculated radiation pattern of array with 30 degree azimuth and elevation angle steering.

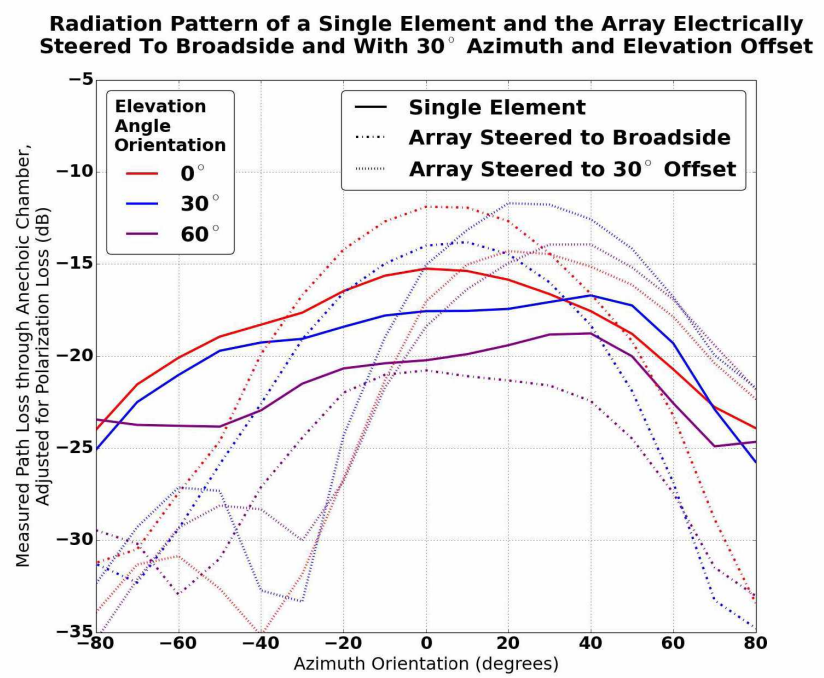

Figure 11. Radiation pattern of array and a single element with a $5 \mathrm{~W}$ power budget.

axis compared to a calculated array pattern with the same electrical steering.

At a 30 degree azimuth and elevation angle mechanical orientation, the measured and calculated path losses agree to within $.5 \mathrm{~dB}$. As expected, the main lobe of the phased array radiation pattern is centered near the 30 degree azimuth and elevation angle orientation.

The difference in the path loss through an anecohic chamber between the phased array and a single element is used to evaluate the performance of the array. Figure 11 shows slices of the radiation pattern of a single antenna element compared to the phased array with the array steered broadside and with a 30 degrees azimuth and elevation offset across azimuth and elevation angle mechanical orientations. The radiation patterns from the array are compensated for polarization loss and a $100 \mathrm{~mW}$ per element overhead with a $5 \mathrm{~W}$ power budget using equations 3 and 4. 
Figure 11 illustrates antenna gain from using the phased array steered broadside and steered with a 30 degrees azimuth and elevation offset. When the array is steered broadside and oriented with no azimuth or elevation angle offsets relative to the receive antenna, the calculated path loss including array overhead is $3.2 \mathrm{~dB}$ lower than the path loss for an individual element with a $5 \mathrm{~W}$ power budget. Likewise, when the array is mechanically oriented with a 30 degree azimuth and 30 degree elevation angle offset, the radiation pattern from the array steered to compensate for the orientation has $5.5 \mathrm{~dB}$ lower path loss than an individual element.

Figure 12 computes this gain across azimuth orientations from -60 degrees to 60 degrees and elevation angle offsets of up to 60 degrees. See Figure 12 for the calculated array gain relative to a single antenna with the same power budget at $2.485 \mathrm{GHz}$ with a simulated fixed power budget of 5 $\mathrm{W}$. The array gain at broadside and one offset illustrated in Figure 11 are marked in Figure 12 with orange dots. The antenna gain measurements are calculated by comparing path loss of single antenna measurements with the path loss of the four element array electrically steered to compensate for mechanical orientation and adjusted for array overhead using Equation 4. This figure illustrates the benefit to EIRP from using the four element phased array on a satellite with a fixed power budget across azimuth and elevation orientations of the array. The array provides an average of $5 \mathrm{~dB}$ gain compared to a single element, with a minimum of $2.5 \mathrm{~dB}$ at -60 degrees azimuth and 10 degrees elevation angle orientation. The degraded performance at high azimuth offsets from broadside could be caused by imperfections in the radiation patterns of the elements.

The gain at some extreme azimuth and elevation steerings exceeds $6 \mathrm{~dB}$, which is the highest expected value from the four element array. This is possibly the result of variation between the radiation patterns of individual elements in the array.

\section{Conclusion}

This paper presented the design of a four element prototype phased array suitable for CubeSat-scale satellites. The array maintains a minimum of $2.5 \mathrm{~dB}$ and average of $5 \mathrm{~dB}$ in EIRP gain compared to a single omnidirectional element up to 60 degrees from broadside with a $5 \mathrm{~W}$ power budget at 2.485 GHz. In the average case, with a $100 \mathrm{~mW}$ per element overhead and 25\% PAE amplifiers, the 4 element phased array consuming only $2 \mathrm{~W}$ could match the EIRP of a single antenna system consuming $5 \mathrm{~W}$.

The prototype T/R modules could scale to 16 elements on a pair of $2 \mathrm{U}$ panels with a $10 \mathrm{~W}$ power budget. Such an array is expected to provide a $11 \mathrm{~dB}$ increase in EIRP compared to a system with a single patch antenna and the same power budget despite $1.6 \mathrm{~W}$ of DC power overhead from the phased array. Phased arrays have the potential to increase the efficiency of CubeSat downlink communication at the expense of mechanical and electrical complexity.

The current prototype is limited to the electronics; mechanical design has been considered only briefly. Future work could include integrating the prototype with a nanosatellite design with an appropriate feed network and cable routing for the control bus. Thermal and vacuum testing of the modules to investigate the robustness of the electronics and array calibration is also needed.

\section{ACKNOWLEDGMENTS}

The research was carried out at the University of Alaska Fairbanks, College of Engineering and Mines, funded in part by the Alaska Space Grant Program.

\section{REFERENCES}

[1] Tethers Unlimited, "SWIFT Software Defined Radios", http://www.tethers.com/SpecSheets/ Brochure_SWIFT_SDR.pdf

[2] Innoflight, "Flight-Heritage S-Band CubeSat Radio", http://innoflight.com/s-band_cubesat_radio/

[3] Iwami, R.T.; Bao Jun Lei; Lim, T.; Tonaki, W.G.; Chun, T.F.; Zamora, A.; Longhi, J.C.; Watanabe, M.K.; Akagi, J.M.; Shiroma, W.A., "Miniaturization challenges for autonomously phased arrays in a CubeSat application," Wireless Information Technology and Systems (ICWITS), 2010 IEEE International Conference on , vol., no., pp.1,4, Aug. 28 2010-Sept. 32010

[4] B. Klofas, "A Survey of CubeSat Communication Systems: 2009-2012," in CubeSat Developers' Workshop, April 2013

[5] B. Klofas, "A Survey of CubeSat Communication Systems," in CubeSat Developers' Conference, April 2009

[6] King, B., "Lockheed Martin CubeSats Studies", http:/www.lockheedmartin.com/content/dam/lockheed/ data/ space/documents/tradeshows/smallsat2012/ 2012\%20CubeSat\%20Fact\%20Sheet_Rev4.pdf

[7] A. W. Rudge; K. Milne; A. D. Olver; P. Knight, "The Handbook of Antenna Design, Volume 2", 1983

[8] Godara, L., "The effect of phase-shifter errors on the performance of an antenna-array beamformer," Oceanic Engineering, IEEE Journal of , vol.10, no.3, pp.278,284, Jul 1985

[9] Forman, B., "Directivity characteristics of scannable planar arrays," Antennas and Propagation, IEEE Transactions on, vol.20, no.3, pp.245,252, May 1972

[10] Benhring Pike, "Air Force Test Range Technical Report AF WTR-TR-65-1", 1965 


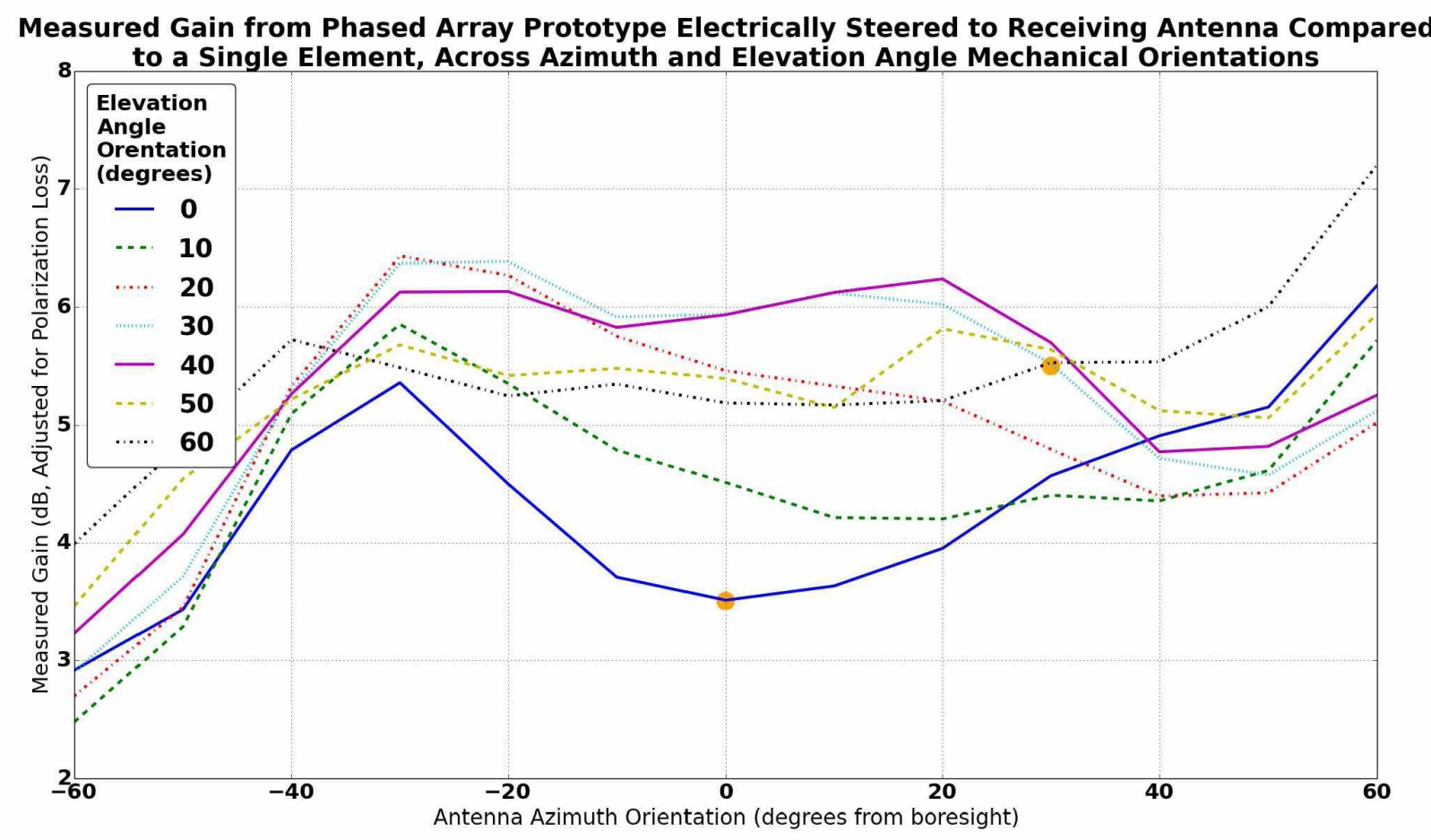

Figure 12. Gain from the 4-element phased array prototype relative to a single patch antenna element with the same power budget across azimuth and elevation steering. The gain from the two array radiation patterns in Figure 11 are marked with orange dots.

\section{BIOGRAPHY}

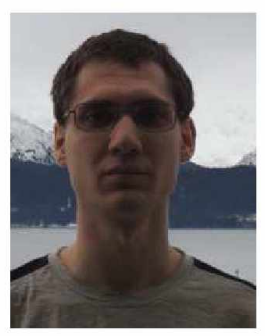

Jonathan Klein received his B.S. (2011) in computer engineering from RoseHulman Institute of Technology. He is currently a graduate student studying electrical engineering at the University of Alaska, Fairbanks.

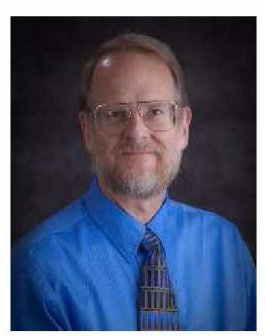

Joe Hawkins received his B.S. (1982) in electrical engineering and mathematics from University of Alaska Fairbanks, and his M.S. (1984) and Ph.D.(1988) from Stanford University. He is currently a Professor of Electrical and Computer Engineering at the University of Alaska Fairbanks.

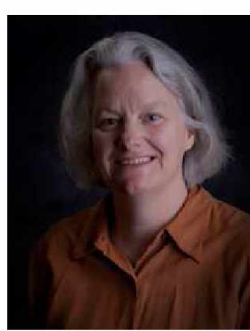

Denise Thorsen received her B.S. (1985), M.S. (1991) and Ph.D. (1996) degrees in electrical and computer engineering from the University of Illinois at Urbana-Champaign. She is an Associate Professor in Electrical and Computer Engineering at the University of Alaska Fairbanks. 